\title{
Surgical approach for Siewert type II adenocarcinoma of the esophagogastric junction: transthoracic or transabdominal? -a single-center retrospective study
}

\author{
Zi-Feng Yang", De-Qing Wü, Jun-Jiang Wang, Xing-Yu Feng, Jia-Bin Zheng, Wei-Xian Hu, Yong Li \\ General Surgery Department, Guangdong General Hospital, Guangdong Academy of Medical Sciences, Guangzhou 510080, China \\ Contributions: (I) Conception and design: ZF Yang, Y Li; (II) Administrative support: None; (III) Provision of study materials or patients: None;(IV) \\ Collection and assembly of data: All authors; (V) Data analysis and interpretation: All authors; (VI) Manuscript writing: All authors; (VII) Final \\ approval of manuscript: All authors. \\ \#These authors contributed equally to this work. \\ Correspondence to: Professor Yong Li, MD, PhD. Guangdong General Hospital, Guangdong Academy of Medical Sciences, Guangzhou 510080, \\ China. Email: yuan821007@126.com.
}

\begin{abstract}
Background: The surgical approach (transthoracic or transabdominal) for patients with Siewert type II adenocarcinoma of the esophagogastric junction (AEG) still remains controversial.

Methods: Data of patients with Siewert type II AEG were collected in the Guangdong General Hospital from 2004 to 2014 and we compared their clinicopathological outcome and prognosis in regard to the transthoracic (TT) and transabdominal (TA) approach.

Results: A total of 158 patients with Siewert type II AEG were analyzed and our results demonstrated that their overall medium survival was 52 months. Also, their 5 -year overall survival rate was 39.1\%, which was comparable between the TT and TA group (35.1\% vs. $43.2 \%, \mathrm{P}>0.05)$, while more lymph nodes were dissected in TA group $(23.7 \pm 0.2$ vs. $18.1 \pm 0.3, \mathrm{P}<0.05)$, with less postoperative complications $(14.3 \%$ vs. $28.4 \%, \mathrm{P}<0.05)$ and shorten hospital stay $(12 \pm 4$ vs. $15 \pm 7 \mathrm{~d}, \mathrm{P}<0.05)$.

Conclusions: For patients with Siewert type II AEG, the TA approach is more suitable to achieve an optimal extent of lymph node dissection, reduction in the incidence of complication, shorten hospital stay, and to promote the recovery.
\end{abstract}

Keywords: Adenocarcinoma of the esophagogastric junction (AEG); Siewert type II; surgical approach

Submitted May 30, 2018. Accepted for publication Oct 16, 2018.

doi: $10.21037 / \mathrm{atm} .2018 .10 .66$

View this article at: http://dx.doi.org/10.21037/atm.2018.10.66

\section{Introduction}

The incidence and mortality of esophageal and gastric cancer is alarming in China and since the past decades, the incidence of adenocarcinoma of the esophagogastric junction (AEG) have been in a progressive increasing trend (1). The classification of AEG proposed by Siewert and Stein is the most common classification used in the present clinical practice and it is mainly based on the location of the epicenter of the carcinoma within a $5 \mathrm{~cm}$ distance above and below the dentate line which demarcates the esophagus from the stomach (2) (Figure 1).
The Union for International Cancer Control (UICC) has suggested that AEG can be categorized using the standards of gastric cancer or esophageal cancer (1). However, there are a variety of differences existing between these two types of cancers regarding their epidemiology, diagnosis, and prognosis and as such there have been different surgical approaches proposed (2,3). According to the Japanese gastric cancer treatment guideline, the transabdominal approach is recommended for AEG if the tumor invasion of the lower esophagus is less than $3 \mathrm{~cm}$. The lymphadenectomy should be performed till be to a certain extent, which indicates the 


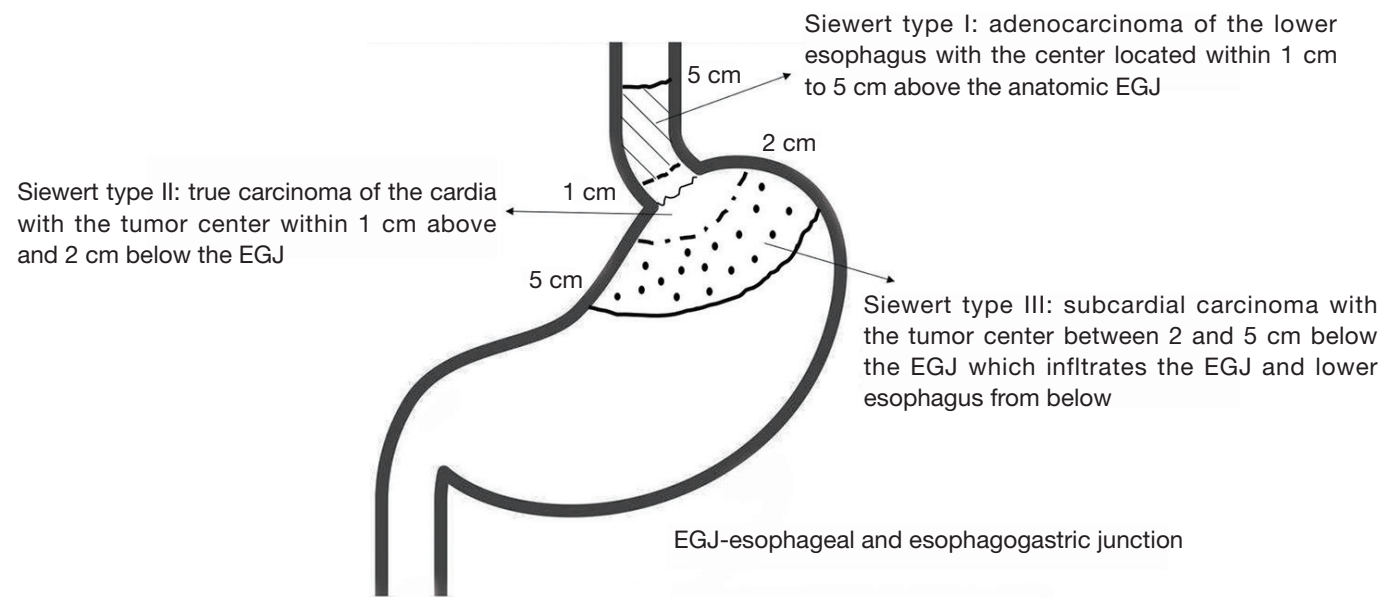

Figure 1 Illustration of the Siewert classification of adenocarcinoma of the esophagogastric junction. The different Siewert types: I, the epicenter of the tumor is located from $1-5 \mathrm{~cm}$ above the dentate line (true esophageal cancers); II, epicenter of the tumor is $1 \mathrm{~cm}$ above and $2 \mathrm{~cm}$ below the dentate line, and; III, the epicenter of the cancer is located $2-5 \mathrm{~cm}$ below the dentate line.

treatment of Siewert type II/III AEG needs referring to gastric cancer model (4). In the National Comprehensive Cancer Network (NCCN) guideline, Siewert type I/II and type III AEG were included in both the esophageal and gastric cancer guideline (5). Therefore, it can be presumed that it has been agreed that Siewert type I and III tumors can be operated transthoracically as performed for those with esophageal cancers or transabdominally as performed for those with gastric cancers. However, there is still controversy regarding the surgical approach for Siewert type II AEG. In this study, the different surgical approach for Siewert type II AEG was investigated by analyzing the clinical data retrospectively.

\section{Methods}

All the patients provided signed consent for participation and this study has received the approval from the Guangdong General Hospital ethical board.

\section{Patients}

Patients with Siewert type II AEG were enrolled from 2004-2014 in Guangdong General Hospital. The inclusion criteria were: (I) Siewert type II AEG; (II) only TT or TA approaches with total or proximal gastrectomy; (III) no distal metastasis; (IV) no prior neoadjuvant chemo-/ chemoradio-therapy before surgery; (V) R0 gastrectomy. The exclusion criteria were: (I) gastric stump carcinoma and non-epithelial malignant tumors; (II) other malignant diseases. The staging was completed according to tumornode-metastasis (TNM) classification of gastric cancer as described in the $7^{\text {th }}$ edition of the American Joint Committee on Cancer (AJCC).

\section{Surgical procedure}

The transthoracic surgery was performed by thoracic surgeons and the gastrectomy was performed from a lateral incision that was made at the left-side chest intercostals space between the $7^{\text {th }}$ and $8^{\text {th }}$ ribs. Regarding the transabdominal surgery, it was performed by gastrointestinal surgeons, for which the gastrectomy was performed from the abdominal midline (Figure 2). Based on the Japanese gastric cancer treatment guidelines (ver. 4) (4), when performing total gastrectomy, all the cases abide to the D2 lymphadenectomy strategy; if tumors invaded the esophagus, the range of lymphadenectomy was extended to cover the posterior mediastinal lymph nodes (station no. 110 and 111), inferior phrenic lymph nodes (station no. 19), diaphragmatic lymph nodes (station no. 20), along with the routinely retrieved perigastric lymph nodes (Figure 3). When performing proximal gastrectomy, all the cases abided to a D1+ lymphadenectomy, i.e., for tumors invading the esophagus, D1+ lymphadenectomy included the retrieval of lymph nodes at station no. 110 (4). For proximal gastrectomy, the main type of anastomosis was esophagogastric anastomosis and for total gastrectomy 
$\pi$

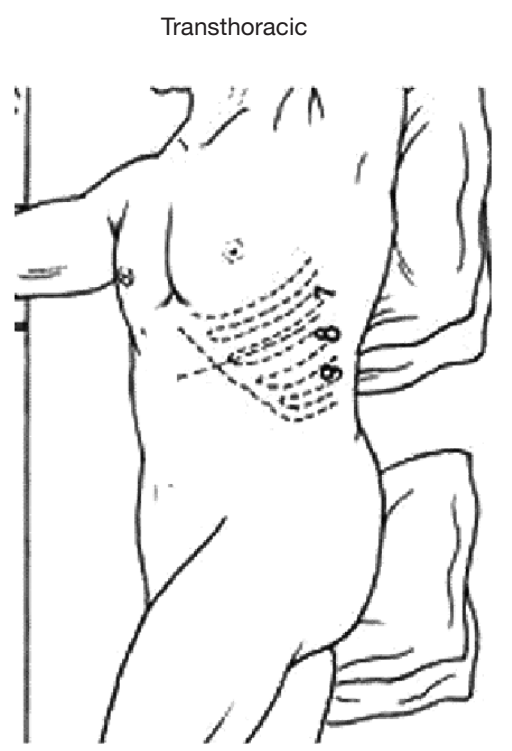

vs.

TA

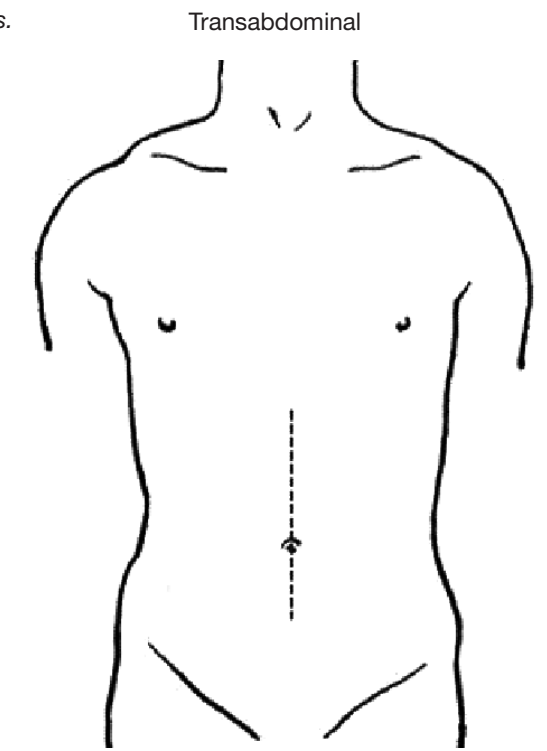

Figure 2 Illustration of the different types of incisions based on transthoracic or transabdominal approach.

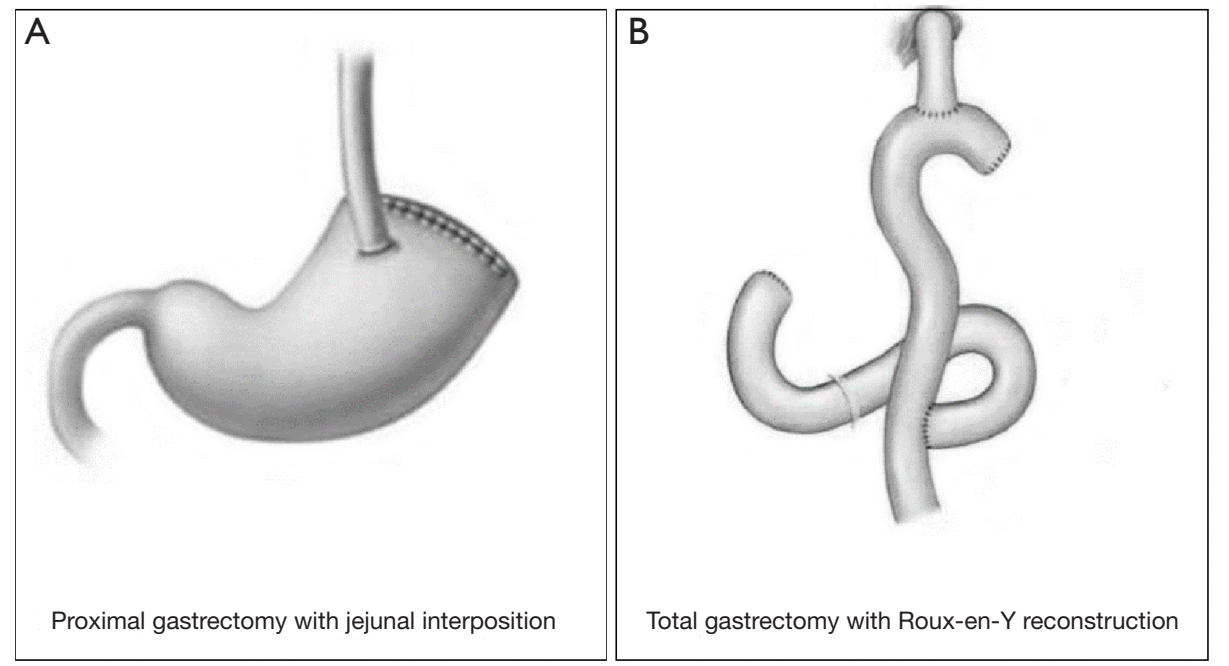

Figure 3 The main type of digestive tract reconstruction performed for (A) proximal gastrectomy, (B) total gastrectomy.

it was the esophageal-jejunum Roux-en-Y anastomosis (Figure 3).

\section{The follow-up observation}

Patients were followed up via phone calls, hospital visits or letters to track the patients' postoperative therapy status, survival status, survival time and quality of life after surgery. The survival time was defined as the time from the surgical day till tumor-related death, or the last day of follow-up. The last follow up day was October 1, 2017.

\section{Statistical analysis}

All statistical analyses were performed using the software SPSS 19.0. Categorical data were compared using the $\mathrm{X}^{2}$ tests or Fisher exact test. Survival curves were derived from the Kaplan-Meier estimates and the curves were compared 
by using the log-rank test. Prognostic factors were identified by univariate analysis and for those factors demonstrating statistical significance $(\mathrm{P}<0.05)$, they were further tested by multivariate analysis. A $\mathrm{P}$ value $<0.05$ was considered as statistically significant.

\section{Results}

\section{Patient characteristics}

A total of 158 cases with Siewert type II AEG were enrolled in accordance with the inclusion criteria, of which, 81 and 77 cases were treated with transthoracic and transabdominal surgery, respectively. Four cases were excluded because they underwent combined thoraco-abdominal surgery. The clinicopathological characteristics of the enrolled patients can be found in Table 1. There were no significant differences observed in regards to the investigated clinicopathological characteristics between the TT and TA group $(\mathrm{P}>0.05$ for all characteristic).

\section{Surgical outcome}

The surgery-related observational characteristics are listed in Table 2. The operative time of the TT group was observed to be significantly longer than that of the TA group $(215 \pm 39$ vs. $168 \pm 26$ min, respectively; $\mathrm{P}<0.05)$. Also, significant differences in the type of surgical resection [greater number of cases of proximal gastrectomy (TT vs. TA, 73 vs. 27, respectively)], number of lymph nodes retrieved (TT vs. TA, $18.1 \pm 0.3$ vs. $23.7 \pm 0.2$, respectively), and the duration of hospitalization (TT $v s$. TA, $15 \pm 7 v s$. $12 \pm 4$, respectively) was observed between the two groups (all $\mathrm{P}<0.05)$. However, no significant difference was observed in the number of positive lymph nodes and the status of the proximal margin between the two groups. Of note, the curative resection rate (R0 gastrectomy) between the TT and TA groups was $95.1 \%$ and $93.5 \%$, respectively $(\mathrm{P}=0.324)$.

\section{Morbidity and mortality}

The postoperative-related observations in terms of morbidity and mortality are listed in Table 3. The rate of postoperative complications (grade $\geq$ II based on the Clavien-Dindo grading system) was observed to be nearly twice in the TT group (28.4\%) as compared to the TA group $(14.3 \%)(\mathrm{P}<0.05)$. The most common complications observed in the TT group was pneumonia $(\mathrm{n}=11 ; 47.8 \%)$ and pleural effusion $(\mathrm{n}=5 ; 21.7 \%)$ while in the TA group it was mostly pneumonia $(n=4 ; 36.4 \%)$. Despite such observations, there was no statistical difference in terms of the 30-day postoperative mortality between the two groups $(\mathrm{P}=0.324)$, although 2 cases and 1 case in the TT and TA group died during that time period.

\section{Survival and prognostic factors}

The medium overall survival (OS) of the TT group, TA group, and the entire study cohort was 36, 51 and 42 months, respectively. No significant difference in terms of survival time between the two groups was observed $(\mathrm{P}=0.278)$. The 5 -year OS rate for the TT and TA group was $35.1 \%$ and $43.2 \%$, respectively (Figure 4). Univariate analysis showed that the age at diagnosis, depth of tumor invasion ( $\mathrm{T}$ stage) $(\mathrm{P}=0.000)$, number of lymph node metastasis ( $\mathrm{N}$ stage) $(\mathrm{P}=0.000)$, TNM stage $(\mathrm{P}=0.000)$, histological differentiation $(\mathrm{P}=0.024)$, vascular tumor emboli $(\mathrm{P}=0.022)$, tumor size $(\mathrm{P}=0.005)$, number of lymphadenectomy $(\mathrm{P}=0.027)$, and adjuvant chemotherapy $(\mathrm{P}=0.012)$ were the main clinicopathological factors affecting prognosis. On multivariate analysis, $\mathrm{N}$ stage was identified as the only independent factor affecting prognosis (Table 4).

\section{Discussion}

AEG is a special type of malignant tumor whose infiltration and invasion may spread across both the esophagus and the stomach. Its surgical treatment is often challenging and usually has a poor prognosis (1). Previous studies have demonstrated that the biological behavior and pathological features of AEG were completely different from those of gastric and esophageal cancer. Siewert type I AEG has been found to be more common in European countries, while type II and III are more common in Asian countries $(1,2)$. According to NCCN guideline, the therapeutics of Siewert type I/II AEG has been classified as to that of esophageal carcinoma, and per se, the transthoracic approach is considered as the recommended surgical procedure (5). However, based from the results obtained from several Asian studies and according to the Japanese gastric cancer treatment guideline (4-8), the biological behavior and pathological features of Siewert type II and III AEG in Asian patients bear more resemblance to gastric cancer than that of esophageal cancer. From the current 
Table 1 Correlation of the clinicopathological characteristics of the 158 Siewert type II AEG patients between the TT and TA group

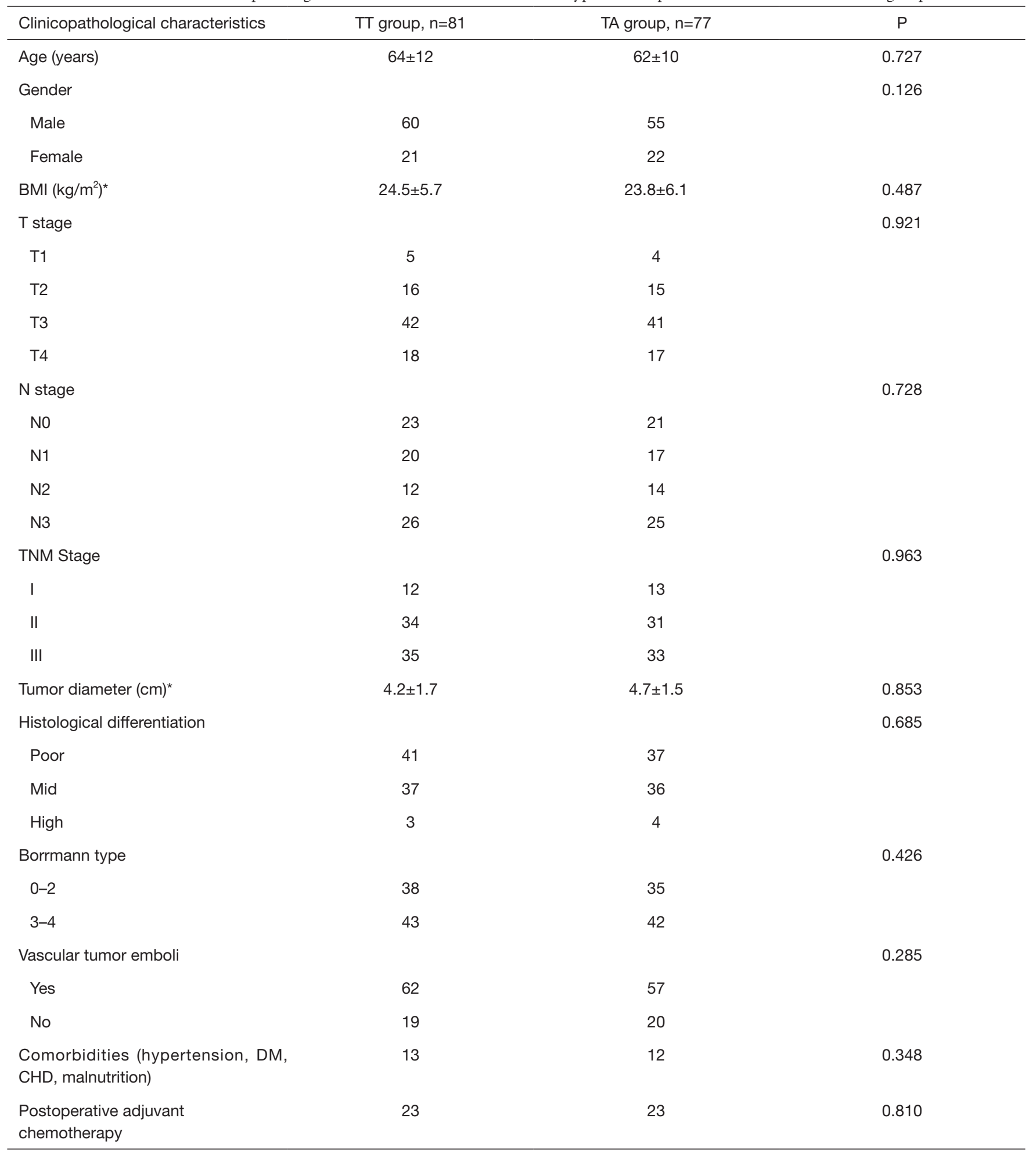

*, for median \pm SD. TT, transthoracic; TA, transabdominal; AEG, adenocarcinomas of the esophagogastric junction; BMI, body mass index; T, depth of tumor invasion; N, metastatic lymph node classification; DM, diabetes; CHD, coronary heart disease. 
Table 2 Comparison of the different surgery-related observational characteristics between the TT and TA group of Siewert type II AEG patients

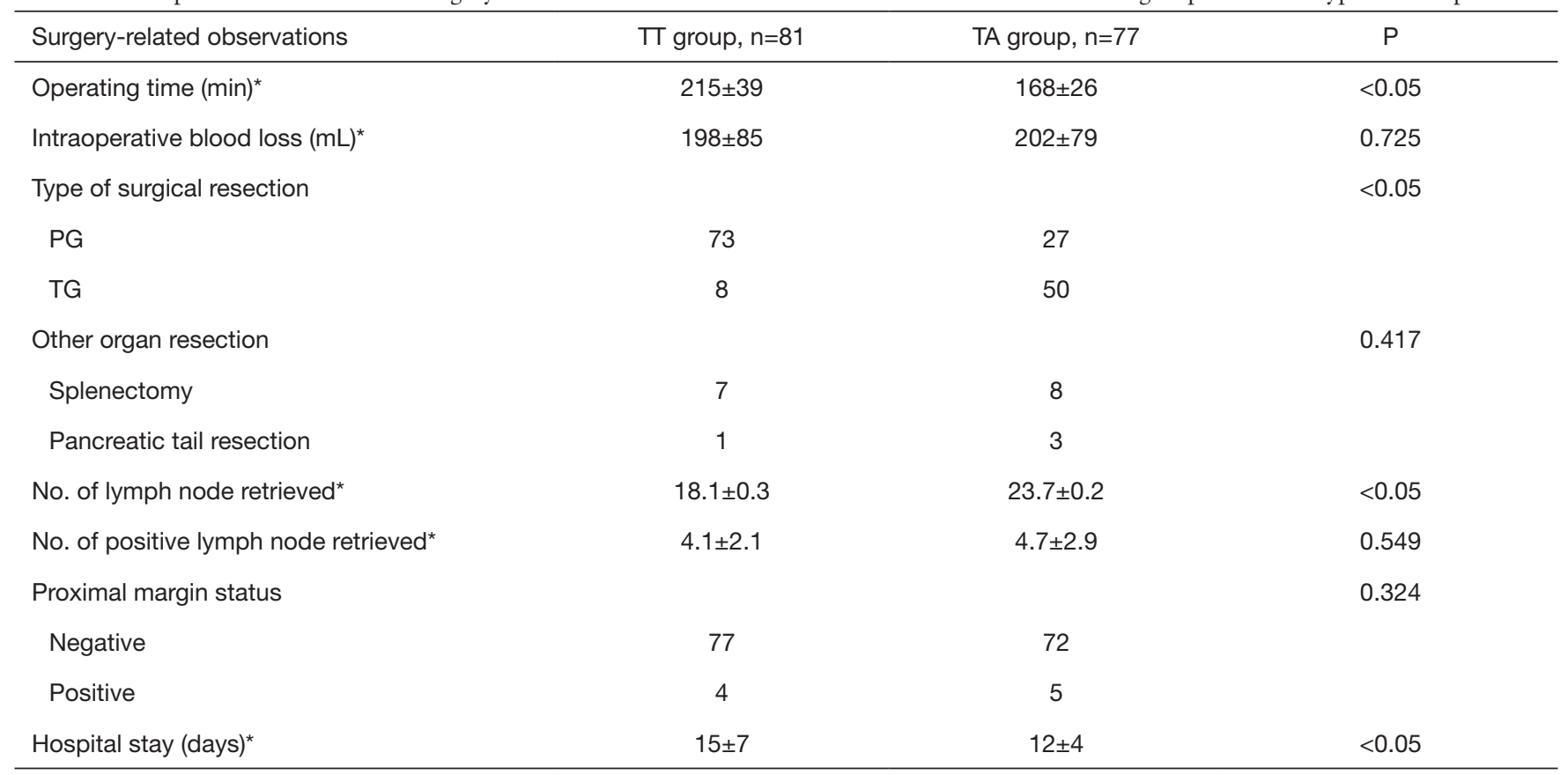

*, for median \pm SD. AEG, adenocarcinomas of the esophagogastric junction; TT, transthoracic; TA, transabdominal; PG, proximal gastrectomy; TG, total gastrectomy.

Table 3 Comparison of the different postoperative morbidity and mortality characteristics between the TT and TA group of Siewert type II AEG patients

\begin{tabular}{|c|c|c|c|}
\hline Postoperative-related observations & TT group, $\mathrm{n}=81$ & TA group, $n=77$ & $\mathrm{P}$ \\
\hline Pneumonia & 11 & 4 & \\
\hline Pleural effusion & 5 & 2 & \\
\hline Abdominal infection & 1 & 1 & \\
\hline Anastomotic leak & 2 & 2 & \\
\hline Pancreatic fistula & 1 & 1 & \\
\hline Postoperative mortality & 2 & 1 & 0.324 \\
\hline
\end{tabular}

*, Classified according to the Clavien-Dindo grading system. AEG, adenocarcinomas of the esophagogastric junction; TT, transthoracic;

TA, transabdominal.

studies (3), for Asian populations, it considered that Siewert type I AEG tumors are to be treated as lower esophageal cancer and the transthoracic approach with additional mediastinal lymph node dissection should be considered, while Siewert type III AEG tumors are to be considered as upper gastric cancers and the surgical treatment should comprised of total gastrectomy alongside with D2 lymphadenectomy (4). However, the treatment of Siewert type II AEG, in regard to the selection of surgical approach and lymph node dissection, is still a controversial dilemma and is worthy for further exploration.

Previous literatures (3-6) have shown that the 


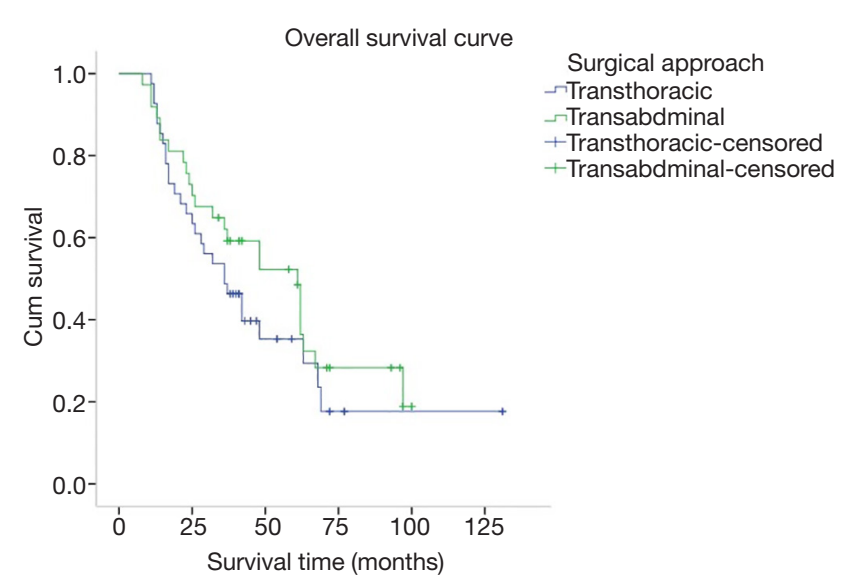

Figure 4 Illustration of the survival curves based on the different surgical approaches of the Siewert type II AEG patients. The 5 -year OS rate for the TT and TA group was $35.1 \%$ and $43.2 \%$, respectively.

transthoracic and transabdominal approach were the most common surgical approaches for Siewert type II AEG and their benefits were also compared. The results showed that the thoraco-abdominal approach not only failed to improve the long-term survival of the patients, but also increased the perioperative complications and mortality. However, the Dutch study revealed that Siewert type II AEG patients treated with transthoracic approach had significantly lower 5 -year overall survival rate than those with transabdominal surgical approach (9). Further, in the JCOG9052 phase III trial conducted by Sasako and colleagues (10), the survival of 167 AEG patients which included 95 Siewert type II patients were investigated in contrast to their surgical approached. Although their results demonstrated no statistical difference between the 2 groups $(\mathrm{P}=0.278)$, the survival curves tend to illustrate a survival advantage for the TA group; which was similar with the results of Zhang' (11) and Zheng' (12) study. Similarly, in this study, upon comparing the long-term survival of Siewert type II AEG patients treated with transthoracic and transabdominal surgical approach, our subgroup analyses indicated that there were no significant differences in survival rate between the TT and TA group but the TA had a tendency to have a better 5 -year OS rate as compared to that of the TT group (43.2\% vs. 35.1\%, respectively; Figure 4). However, due to the risky nature of the location of such tumors, the surgeries are recommended to be performed cautiously (8) and in centers with great expertise.

Currently, the extent of lymph node dissection for patients with Siewert type I and III AEG has been recognized as an important factor affecting the prognoses by most experts. However, regarding Siewert type II AEG patients, their lymph node metastases tend to reside in both the chest and the abdomen. Thus, many controversies and difficulties are present in their surgical treatment $(13,14)$. Numerous studies (15-17) have indicated that the lymph nodes metastasis of Siewert type II AEG has the potential to transfer from the abdomen to the chest, but they are still mainly found in the abdominal perigastric nodes (particularly in stations number 1, 2, 3 and 7). Although theoretically, the thoraco-abdominal approach would provide a higher yield of lymph node retrieval, however, it is not recommended in clinical practice due to the lower metastasis rate of thoracic lymph node and an increased risk of complication $(18,19)$. Meanwhile, many investigators have demonstrated that the transabdominal approach as a more favorable approach for performing abdominal lymph node dissection than the thoracic approach since more lymph nodes can be harvested and result in a more satisfactory survival outcome. Thus, the lymph node dissection via transabdominal approach was recommended (20-23). In our study, we found that the number of lymph nodes dissection in the transabdominal approach was significantly higher than that of the transthoracic approach $(23.7 \pm 0.2$ vs. $18.1 \pm 0.3, \mathrm{P}<0.05)$. This result is consistent with previous reports $(11,12)$ and may explain the survival benefits of the transabdominal approach in these studies. In contrast, in our study, although the number of lymph nodes harvested using the TA approach was higher, however, no difference in survival between the TT and TA group was observed and we presume that it was possibly because there were no difference in the number of metastatic lymph nodes between these two approaches was observed $(4.1 \pm 2.1$ vs. $4.7 \pm 2.9$, respectively; $\mathrm{P}=0.549)$. In the study of Goto et al. (19), AEG lymph nodes metastasis was mainly abdominal metastasis and concentrated in stations 1, 2, 3, 7, 9 and 19, which are nearby the proximal part of the stomach, among which, the rate of metastasis for station 1 and 3 were higher (37.0-52.5\%), while the metastatic rates of the distal stations 4, 5 and 6 were low. As such, considering that the status of the proximal margin was also similar between both the TA and TT groups in this study $(\mathrm{P}=0.324)$, our data demonstrate that both approaches can meet the radical standard for lymph node dissection and gastrectomy.

The risk of complication is an important factor that surgeons consider upon selecting the optimal surgical 
Table 4 Univariate and multivariate analyses of 158 Siewert type II AEG patients for overall survival

\begin{tabular}{|c|c|c|c|c|}
\hline Variables & \multicolumn{2}{|c|}{ Univariate analysis } & \multicolumn{2}{|c|}{ Multivariate analysis } \\
\hline Age & $1.127(0.561-2.26)$ & 0.037 & $1.210(0.761-1.942)$ & 0.543 \\
\hline Gender & $0.733(0.361-1.48)$ & 0.897 & & \\
\hline Other organ resection & $0.678(0.456-1.287)$ & 0.887 & & \\
\hline Surgical resection way & $0.728(0.364-1.455)$ & 0.181 & & \\
\hline Blood loss & $1.271(0.644-2.507)$ & 0.608 & & \\
\hline T stage & $6.897(3.190-14.911)$ & 0.000 & & \\
\hline $\mathrm{N}$ stage & $3.061(2.078-4.510)$ & 0.000 & $2.247(1.820-2.775)$ & 0.000 \\
\hline Tumor size & $1.107(0.491-2.495)$ & 0.005 & $1.311(0.814-2.112)$ & 0.345 \\
\hline Histological differentiation & $1.349(0.765-2.378)$ & 0.024 & $0.836(0.584-1.195)$ & 0.273 \\
\hline Vascular tumor emboli & $1.094(0.575-2.082)$ & 0.022 & $1.661(1.021-2.732)$ & 0.175 \\
\hline Hospitalization days & $0.902(0.470-1.730)$ & 0.099 & & \\
\hline Chemotherapy & $0.796(0.402-1.576)$ & 0.012 & $0.752(0.482-1.171)$ & 0.327 \\
\hline
\end{tabular}

approach because this will determine the recovery of the patients, their hospital stay, cost of hospitalization, the time for adjuvant chemotherapy. The Dutch study and the JCOG9502 clinical trial demonstrated that for Siewert type II AEG patients, the incidence of postoperative complications such as cardiac dysfunction, anastomotic and chylous leakage, and prolonged hospitalization were significantly increased in TT group than those in the TA group $(9,10)$. Similarly, a meta-analysis including 16 studies indicated that significantly higher incidence of cardiovascular and respiratory complications, and longer length of hospital stay were observed in the TT group (12). The results in our study demonstrate consistency with those previous literatures as the incidence of postoperative complications in the TT group was significantly higher than that in the TA group $(\mathrm{P}<0.05)$.

Compared to previous literatures, although no survival benefits between the TT and TA groups was observed in terms of statistics, the limitations of this study is worth mentioning. We believe that the retrospective nature of this single-center study and the limited pool of data used for analysis, to some extent, may have some effects on the results obtained. Therefore, prospective and multi-centered studies involving a large sample of data are still required to validate the results of this study.

\section{Conclusions}

Our results demonstrated that there was no survival difference for Siewert type II AEG patients upon opting between the transthoracic or transabdominal surgical approach. However, our results showed that less lymph node was resected as a higher incidence of postoperative complications and a longer length of hospitalization arised in TT group. Nonetheless, we recommend the transabdominal approach to treat Siewert type II AEG since it can achieve a greater radicality of lymphadenectomy, lower risk of postoperative complications and shorten hospitalization stay.

\section{Acknowledgements}

Funding: This study is supported by Natural Science Foundation of Guangdong Province (2016A030313762). Then, Dr. ZF Yang would like to acknowledge the support and guidance received from his postgraduate mentor, Prof. 
$\mathrm{Y} \mathrm{Li}$ in the past 4 years.

\section{Footnote}

Conflicts of Interest: The authors have no conflicts of interest to declare.

Ethical Statement: This study was approved by the Research Ethics Committee, Guangdong General Hospital, Guangdong Academy of Medical Sciences, Guangzhou, China (No. 2016296H). Informed consent for participation in the study was obtained either directly, or from a guardian of each patient.

\section{References}

1. Hasegawa S, Yoshikawa T. Adenocarcinoma of the esophagogastric junction: incidence, characteristics, and treatment strategies. Gastric Cancer 2010;13:63-73.

2. Siewert JR, Stein HJ. Classification of adenocarcinoma of the oesophagogastric junction. Br J Surg 1998;85:1457-9.

3. Deng JY, Liang H. Adenocarcinoma of esophagogastric junction. Chin J Cancer Res 2014;26:362-3.

4. Japanese Gastric Cancer Association. Japanese gastric cancer treatment guidelines 2014 (ver. 4). Gastric Cancer 2017;20:1-19.

5. Ajani JA, D'amico TA, Almhanna K, et al. Gastric Cancer, Version 3.2016, NCCN Clinical Practice Guidelines in Oncology. J Natl Compr Canc Netw 2016;14:1286-312.

6. Suh YS, Kong SH, Lee HJ, et al. Reply to the Letter: "Should Adenocarcinoma of the Esophagogastric Junction Be Classified as Gastric or Esophageal Cancer, or Else as a Distinct Clinical Entity?". Ann Surg 2016;264:e5-6.

7. Hasegawa S, Yoshikawa T, Aoyama T, et al. Esophagus or stomach? The seventh TNM classification for Siewert type II/III junctional adenocarcinoma. Ann Surg Oncol 2013;20:773-9.

8. Kurokawa Y, Takiguchi S, Mori M, et al. Surgery for esophagogastric junction tumor. Nihon Shokakibyo Gakkai Zasshi 2015;112:1785-90.

9. Omloo JM, Lagarde SM, Hulscher JB, et al. Extended transthoracic resection compared with limited transhiatal resection for adenocarcinoma of the mid/distal esophagus: five-year survival of a randomized clinical trial. Ann Surg 2007;246:992-1000; discussion 1000-1.

10. Sasako M, Sano T, Yamamoto S, et al. Left thoracoabdominal approach versus abdominal-transhiatal approach for gastric cancer of the cardia or subcardia: a randomised controlled trial. Lancet Oncol 2006;7:644-51.

11. Zhang W, Chen X, Liu K, et al. Comparison of survival outcomes between transthoracic and transabdominal surgical approaches in patients with Siewert-II/III esophagogastric junction adenocarcinoma: a singleinstitution retrospective cohort study. Chin J Cancer Res 2016;28:413-22.

12. Zheng Z, Cai J, Yin J, et al. Transthoracic versus abdominal-transhiatal resection for treating Siewert type II/III adenocarcinoma of the esophagogastric junction: a meta-analysis. Int J Clin Exp Med 2015;8:17167-82.

13. Peng J, Wang WP, Yuan Y, et al. Optimal Extent of Lymph Node Dissection for Siewert Type II Esophagogastric Junction Adenocarcinoma. Ann Thorac Surg 2015;100:263-9.

14. Schuhmacher C, Novotny A, Feith M, et al. The new TNM classification of tumors of the esophagogastric junction. Surgical consequences. Chirurg 2012;83:23-30.

15. Saba N F. Esophagogastric junction and gastric adenocarcinoma: current challenges and future directions. Oncology (Williston Park) 2014;28:520-1.

16. Sisic L, Blank S, Weichert W, et al. Prognostic impact of lymph node involvement and the extent of lymphadenectomy (LAD) in adenocarcinoma of the esophagogastric junction (AEG). Langenbecks Arch Surg 2013;398:973-81.

17. Yamashita H, Katai H, Morita S, et al. Optimal extent of lymph node dissection for Siewert type II esophagogastric junction carcinoma. Ann Surg 2011;254:274-80.

18. Wei MT, Zhang YC, Deng XB, et al. Transthoracic vs transhiatal surgery for cancer of the esophagogastric junction: a meta-analysis. World J Gastroenterol 2014;20:10183-92.

19. Goto H, Tokunaga M, Miki Y, et al. The optimal extent of lymph node dissection for adenocarcinoma of the esophagogastric junction differs between Siewert type II and Siewert type III patients. Gastric Cancer 2014;18:375-81.

20. Chen XZ, Zhang WH, Hu JK. Lymph node metastasis and lymphadenectomy of resectable adenocarcinoma of the esophagogastric junction. Chin J Cancer Res 2014;26:237-42.

21. Blank S, Schmidt T, Heger P, et al. Surgical strategies in true adenocarcinoma of the esophagogastric junction (AEG II): thoracoabdominal or abdominal approach?. Gastric Cancer 2018;21:303-14.

22. Sugita S, Kinoshita T, Kaito A, et al. Short-term outcomes after laparoscopic versus open transhiatal resection of 
Siewert type II adenocarcinoma of the esophagogastric junction. Surg Endosc 2018;32:383-90.

23. Yang $Z$, Wang J, Wu D, et al. Retrospectively analysis of the pathology and prognosis of 131 cases of adenocarcinoma of the esophagogastric junction (Siewert type II/III). Transl Cancer Res 2017;6:949-59.

Cite this article as: Yang ZF, Wu DQ, Wang JJ, Feng XY, Zheng JB, Hu WX, Li Y. Surgical approach for Siewert type II adenocarcinoma of the esophagogastric junction: transthoracic or transabdominal? - a single-center retrospective study. Ann Transl Med 2018;6(23):450. doi: 10.21037/atm.2018.10.66 